\title{
Human complement factor $H$ is a novel diagnostic marker for lung adenocarcinoma
}

\author{
TIANTIAN CUI ${ }^{1}$, YUAN CHEN ${ }^{1}$, THOMAS KNÖSEL ${ }^{1}$, LINLIN YANG $^{1}$, KRISTIN ZÖLLER $^{1}$, \\ KERSTIN GALLER ${ }^{1}$, ALEXANDER BERNDT ${ }^{1}$, MICHAEL MIHLAN ${ }^{2}$, PETER F. ZIPFEL ${ }^{2}$ and IVER PETERSEN ${ }^{1}$ \\ ${ }^{1}$ Institute of Pathology, University Hospital Jena, Friedrich-Schiller-University Jena, \\ Ziegelmühlenweg 1, Jena 07743; ${ }^{2}$ Department of Infection Biology, Leibniz Institute for Natural \\ Product Research and Infection Biology, Beutenbergstr. 11a, 07745 Jena, Germany
}

Received December 10, 2010; Accepted January 24, 2011

DOI: $10.3892 /$ ijo.2011.1010

\begin{abstract}
Human complement factor $\mathrm{H}(\mathrm{CFH})$, a central complement control protein, is a member of the regulators of complement activation family. Recent studies suggested that CFH may play a key role in the resistance of complementmediated lysis in various cancer cells. In this study, we investigated the role of $\mathrm{CFH}$ in human lung cancer. Expression of CFH was analyzed in lung cancer cell lines by RT-PCR, Western blotting and immunofluorescence. In primary lung tumors, the protein expression of $\mathrm{CFH}$ was evaluated by immunohistochemistry (IHC) on tissue microarray (TMA). Binding of $\mathrm{CFH}$ to lung cancer cells was detected by flow cytometry. mRNA expression of CFH was detected in 6 out of 10 non-small cell lung cancer (NSCLC) cell lines, but in none of the small cell lung cancer (SCLC) cell lines. In line with Western blotting, immunofluorescence analysis demonstrated CFH protein expression in 3 NSCLC cell lines, and the immunoreaction was mainly associated with cell cytoplasm and membrane. In primary lung tumors, 54 out of 101 samples exhibited high expression of $\mathrm{CFH}$ and high expression was significantly correlated with lung adenocarcinoma $(\mathrm{p}=0.009)$. Also, in adenocarcinoma of the lung, Kaplan-Meier survival analysis showed a tendency that CFH-positive tumors had worse prognosis in comparison to $\mathrm{CFH}$-negative tumors $(p=0.082)$. Additionally, shorter survival time of patients with adenocarcinoma ( $<20$ months) was associated with higher staining of $\mathrm{CFH}(\mathrm{p}=0.033)$. Our data showed that non-small cell lung cancer cells expressed and secreted $\mathrm{CFH}$. $\mathrm{CFH}$ might be a novel diagnostic marker for human lung adenocarcinoma.
\end{abstract}

Correspondence to: Dr Iver Petersen, Institute of Pathology, University Hospital Jena, Friedrich-Schiller-University Jena, Ziegelmühlenweg 1, 07743 Jena, Germany

E-mail: iver.petersen@med.uni-jena.de

Key words: complement factor $\mathrm{H}$, lung cancer, diagnostic marker, adenocarcinoma, prognosis

\section{Introduction}

Lung cancer has a 5 -year survival rate of $\sim 15 \%$ and is a leading cause of cancer-related deaths. Considerable progress has been made in defining genetic defects of precursor lesions $(1,2)$. However, the advanced carcinoma is the major problem for the clinician. The majority of lung cancers is either not resectable at the time of diagnosis or has already spread to distant organs. Therefore, reliable biomarkers for the early diagnosis and prediction of survival are increasingly demanded.

Lung cancer can be divided into two histological groups. Small cell lung carcinoma (SCLC) comprises approximately $20 \%$ of the total incidence, while non-small cell lung carcinoma (NSCLC), which includes adenocarcinoma, squamous cell carcinoma (SCC) and large cell carcinoma, accounts for $80 \%$ (3). Whereas adenocarcinomas tend to be located in the periphery of the lung, squamous cell carcinomas arise preferentially from bronchi near the hilus with potential involvement of trachea. SCC derives from stem cells of a dysplastic multilayer epithelium that underwent squamous metaplasia, whereas adenocarcinoma originates preferentially from precursor cells of the mono- or bilayer surface epithelium of the lung periphery. For SCC of lung, p63 and Ck5/6 are widely used as diagnostic markers, while thyroid transcription factor-1 (TTF-1) is a marker for lung ADC (4). However, in some cases, even in combination with these markers, SCC and ADC still could not be distinguished from each other. Given that patients with certain subtypes of lung cancer could benefit from chemotherapy or targeted therapy, identification of biomarkers to distinguish different subtypes of lung cancer is becoming increasingly important.

It has been recognized that tumor cells have mechanisms to circumvent the complement-mediated immune response, and human non-small cell lung cancer cells are especially resistant to complement mediated lysis as compared to normal cells (5). The complement system consists of a series of regulatory proteins, which inhibit complement activation by interacting with one another in a cascade manner, leading in consequence to cell lysis (6). The alternative pathway of complement is spontaneously activated and requires specific regulators to restrict the destructive effects of foreign cells 
and microbes or modified self cells (7). CFH is a key inhibitor of the alternative pathway, and it is the prototype of a family of CFH-related molecules (8). The family includes $\mathrm{CFH}$, complement factor H-like protein 1 (FHL-1) and complement factor H-related proteins (CFHRs). Evidence supporting the involvement of $\mathrm{CFH}$ in carcinogenesis is accumulating. For instance, malignant glioblastoma cells produce $\mathrm{CFH}$ and FHL-1 $(9,10)$. Secretion of soluble complement inhibitor CFH and FHL-1 by ovarian tumor cells could protect tumor cells against humoral immune attack and posed an obstacle for therapy with monoclonal antibodies (11). Similarly, in colon and lung cancer, the possible role of $\mathrm{CFH}$ was resistance to complement attack $(6,12)$. Additionally, CFH or related proteins were considered as diagnostic markers for transitional cell cancer of the bladder (13).

In this study, we analyzed the expression of $\mathrm{CFH}$ in a panel of lung cancer cell lines and in primary lung tumors. We found that CFH was expressed in 6 out of 10 of NSCLC cell lines but not in any of SCLC cell lines tested. For the first time, we provide evidence that $\mathrm{CFH}$ might be a diagnostic marker for adenocarcinoma of lung.

\section{Materials and methods}

Cell lines and cell culture. Human lung carcinoma cell lines, including non-small cell lung carcinomas (non-SCLCs: H23, H2030, H2228, H157, H226, H2170 and H322), and small cell lung carcinomas (SCLCs: SHP77, CPC-N, H82, COLO668, COLO677 and H526) were purchased from the American Type Culture Collection (ATCC, Rockville, MD, USA) and from the German Collection of Micro-organisms and Cell Culture (DSMZ, Braunschweig, Germany). Cells were grown in RPMI-1640 medium (Biochrom AG, Germany) supplemented with $10 \%$ fetal bovine serum, and maintained in a humidified atmosphere with $5 \% \mathrm{CO}_{2}$ at $37^{\circ} \mathrm{C}$. In addition, three NSCLC cell lines including D51, D54 and D117 were established in our laboratory. They were derived from primary tumors of patients who were operated at the University Hospital Charité. These cells were grown in Leibovitz 15 media supplemented with $10 \%$ FCS and $1 \%$ glutamine.

$R T-P C R$ analysis. Total RNA was extracted from the cells using the TRIzol reagent (Invitrogen) according to the manufacturer's instructions. Total RNA $(1 \mu \mathrm{g})$ was incubated with RQ1 RNase-free DNase (Promega, USA) for $30 \mathrm{~min}$ at $37^{\circ} \mathrm{C}$ to eliminate genomic DNA contamination.

First-strand cDNA was reverse-transcribed by $15 \mathrm{U}$ ThermoScript RT (Invitrogen) from the DNase-treated RNA in the presence of 1XRT buffer, $100 \mathrm{mMDTT}, 20 \mathrm{U}$ RNase inhibitor, and $10 \mathrm{mM}$ dNTPs, using the random hexamer primers supplied in the kit. PCR amplification of CFH was carried out with the following primer pair: 5'-CTTCCTTGTAAATCTCCACCTG-3' (sense) and 5'-TCTGCATGTTGGCCTTCC TGTC-3' (antisense). PCR was performed under the following condition: $94^{\circ} \mathrm{C} 1 \mathrm{~min}$, $53^{\circ} \mathrm{C} 30 \mathrm{sec}, 72^{\circ} \mathrm{C} 30 \mathrm{sec}$, for 35 cycles, with initial denaturation at $95^{\circ} \mathrm{C} 15 \mathrm{~min}$ and final elongation at $72^{\circ} \mathrm{C} 7 \mathrm{~min}$. Controls were performed with glyceraldehyde-3-phosphate dehydrogenase (GAPDH) primer: 5'-GTGAAGGTCGGAGTCAACG-3' (sense) and 5'-AGGAGGCATTGCTGATGAT-3' (antisense). The expected size of PCR product for CFH is $348 \mathrm{bp}$. The PCR products were visualized on a $1.5 \%$ agarose gel stained with ethidium bromide.

Western blotting. Cells were grown in RPMI-1640 without FCS for $48 \mathrm{~h}$. Supernatants from cells were concentrated 50 -fold using centrifugal filter units (Centricon YM-10, Millipore Corporation, Billerica, MA). Total protein $(20 \mu \mathrm{g})$ was used for Western blotting according to a standard protocol. Monoclonal anti-factor H antibody OX-24 (AbD Serotec, UK) was used at a 1:500 dilution. Signal was visualized with peroxidase-conjugated polyclonal rabbit anti-mouse antibody (1:1000, Dako) and ECL Plus Western blotting Detection System (GE Healthcare, Germany).

Immunofluorescence. Cells were grown on 4-well cell culture slides (Falcon, Germany) in RPMI-1640 without FCS. After $48 \mathrm{~h}$, cell culture slides were fixed in $-20^{\circ} \mathrm{C}$ acetone for $3 \mathrm{~min}$. After air drying, the slides were stored at $-80^{\circ} \mathrm{C}$ or immediately subjected to immunofluorescence.

For immunofluorescence, a monoclonal anti-factor $\mathrm{H}$ antibody OX-24 (AbD Serotec) was used at a 1:25 dilution. Bound antibodies were detected using $\mathrm{Cy} 3$-conjugated goat antimouse IgG (Dianova, Germany, dilution 1:200). Fluorescence labeling was analyzed by confocal laser scanning microscopy (LSM 510, Zeiss, Germany) as described previously (14).

Tissue microarray construction and immunohistochemical analysis. Two hundred and two tumor specimens from 101 patients with primary lung tumor were used for the construction of tissue microarray (TMA). All of these patients were undergoing surgical operation at the Department of Surgery of the University Hospital Charité. No adjuvant radiotherapy or chemotherapy was administered before surgery. The clinicopathological features of the samples are listed in Table I according to the WHO classification of lung cancer. The study was approved by the local ethics committee.

TMA was constructed by using a manual tissue microarray purchased from Beecher Instruments (Woodland, WI, USA). Suitable areas for tissue retrieval were marked on standard haematoxylin and eosin (H\&E) sections. Two tissue cylinders per tumor with a diameter of $0.6 \mathrm{~mm}$ were present in one TMA.

A section from the tissue microarray was dewaxed with xylene and gradually hydrated. Antigen retrieval was performed by treatment in a pressure cooker for $6 \mathrm{~min}$. The mouse antifactor $\mathrm{H}$ monoclonal antibody with working concentration of 1:100 (AbD Serotec) was incubated at room temperature for $1 \mathrm{~h}$. Detection took place according to the manufacturer's instructions (LSAB ${ }^{\mathrm{TM}}$ 2-kits, Dako, Denmark). All slides were read by two pathologists (T. Knösel and I. Petersen) who were blinded to the clinical information. Immunohistochemistry was scored semiquantitatively as negative (score 0 ), weak (score 1), moderate (score 2 ) or strong (score 3 ) as previously described (15). For statistical evaluation, scores 0 was considered as negative, while scores 1,2 and 3 together as positive.

Flow cytometry. Lung cancer cell lines COLO677, COLO668, H2228 and H226, were cultured in RPMI-1640 medium without FCS for $48 \mathrm{~h}$, washed once, and resuspended in $0.5 \mathrm{X}$ PBS containing $1 \%$ BSA (PBS-BSA). Cells were incubated 
A

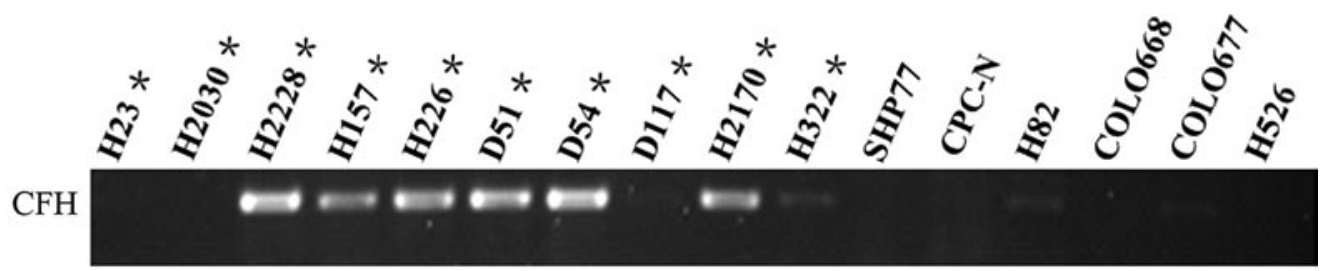

GAPDH

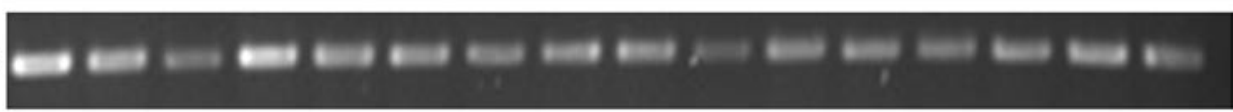

B

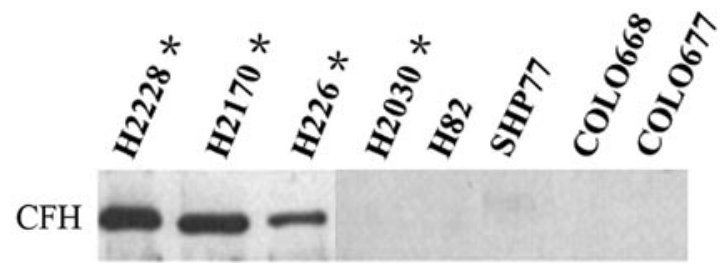

Figure 1. Analysis of CFH expression in lung cancer cell lines by (A) RT-PCR and (B) Western blotting showed that CFH was expressed and secreted in several NSCLC cell lines, but not in SCLC cell lines. NSCLC cell lines are marked with *.

with or without purified human CFH diluted in PBS-BSA for $20 \mathrm{~min}$ at $37^{\circ} \mathrm{C}$. Purified $\mathrm{CFH}$ was used at a concentration of 10 and $20 \mu \mathrm{g} / \mathrm{ml}$. Presence of endogenous CFH on the cells and binding of purified CFH (Complement Technology Inc., TX, USA) to the cells was detected with goat anti-human CFH antibody (Merck Biosciences, Germany, 1:500 in PBS-BSA) and Alexa Fluor 488-labeled rabbit anti-goat IgG (Invitrogen, Germany, 1:800 in PBS-BSA). Goat pre-immune sera (Merck Bioscience) were used as control. Intact DAPI negative cells were analyzed. Amount of $1 \times 10^{4}$ cells were routinely measured in each sample by using a BD LSRII flow cytometer, and FACSDiva Software (BD Biosciences, Heidelberg, Germany). Data evaluation was done with FlowJo (Tree Star, Ashland, OR, USA).

Statistical analysis. To compare the protein expression of $\mathrm{CFH}$ with clinicopathological parameters, $2 \mathrm{x} 2$ contingency tables were set up and Chi-square or Fisher's exact tests was performed. Kaplan-Meier survival curves were constructed for statistical significance with the log-rank tests. Data obtained from CFH binding were analyzed by Student's t-test. All p-values were calculated two-sided. For all tests, $\mathrm{p}<0.05$ was considered statistically significant. The statistical analysis was performed by using the software package SPSS 13.0 (SPSS, Chicago, IL, USA).

\section{Results}

Expression analysis of $\mathrm{CFH}$ in human lung cancer cell lines. RT-PCR was performed to analyse the expression of $\mathrm{CFH}$ in 16 cultured lung cell cancer lines. We found that $\mathrm{CFH}$ was expressed in 6 out of 10 non-small cell lung cancer cell lines, while in 6 small cell lung cancer cell lines tested, there was no signal detectable (Fig. 1A).

To analyse the protein expression of $\mathrm{CFH}$ in lung cancer cell lines, we performed Western blotting in 8 cell lines by using protein from cell culture medium without FCS. We found that protein expression of $\mathrm{CFH}$ was detected in 3 non-small cell lung cancer cell lines, but not in any of small cell lung cancer cell lines (Fig. 1B). These results further demonstrated that $\mathrm{CFH}$ was expressed in some non-small cell lung cancer cell lines but not in small cell lung cancer cell lines, and CFH protein is secreted to extracellular medium.

Immunofluorescence. To verify the results from Western blotting and to evaluate the location of the factor $\mathrm{H}$ protein in lung cancer cell lines, we performed immunofluorescence in 4 lung cancer cell lines including H226, H2228, H2170 and COLO677. As expected, protein expression of $\mathrm{CFH}$ was found in 3 NSCLC cell lines H226, H2228 and H2170 (Fig. 2A-C), but not in the SCLC cell line COLO677 (Fig. 2D). This result is in good agreement with that from Western blotting. Additionally, the immunoreaction was found to be mainly associated with cell cytoplasm and membrane.

Lung cancer cells bind CFH to their surface. To investigate whether tumor cells bind $\mathrm{CFH}$ to the cell surface, 4 lung cancer cell lines were incubated with purified $\mathrm{CFH}$, and $\mathrm{CFH}$ binding was analyzed to intact cells by flow cytometry analysis. The two NSCLC cell lines H2228 and H226 bound endogenous CFH on their cell surface (Fig. 3). In addition, $\mathrm{H} 226$ cells bound purified CFH from the extracellular space in a concentration-dependent manner (Fig. 3). In contrast, the small lung cancer cells COLO677 and COLO668 did not bind endogenous $\mathrm{CFH}$ to the cell surface, but attached exogenous $\mathrm{CFH}$ in a concentration-dependent manner (Fig. 3). Binding of exogenous CFH to H226, COLO677 and COLO668 was confirmed using human serum (data not shown). These results show that non-small lung cancer cells, which produce and secret $\mathrm{CFH}$, attach $\mathrm{CFH}$ to their cell surface. Additionally, small cell lung cancer cells, which do not produce $\mathrm{CFH}$, bind $\mathrm{CFH}$ from extracellular space sources like plasma or other body fluid.

Immunohistochemical analysis of $\mathrm{CFH}$ in primary lung tumors. Tissue microarray was constructed to explore $\mathrm{CFH}$ 

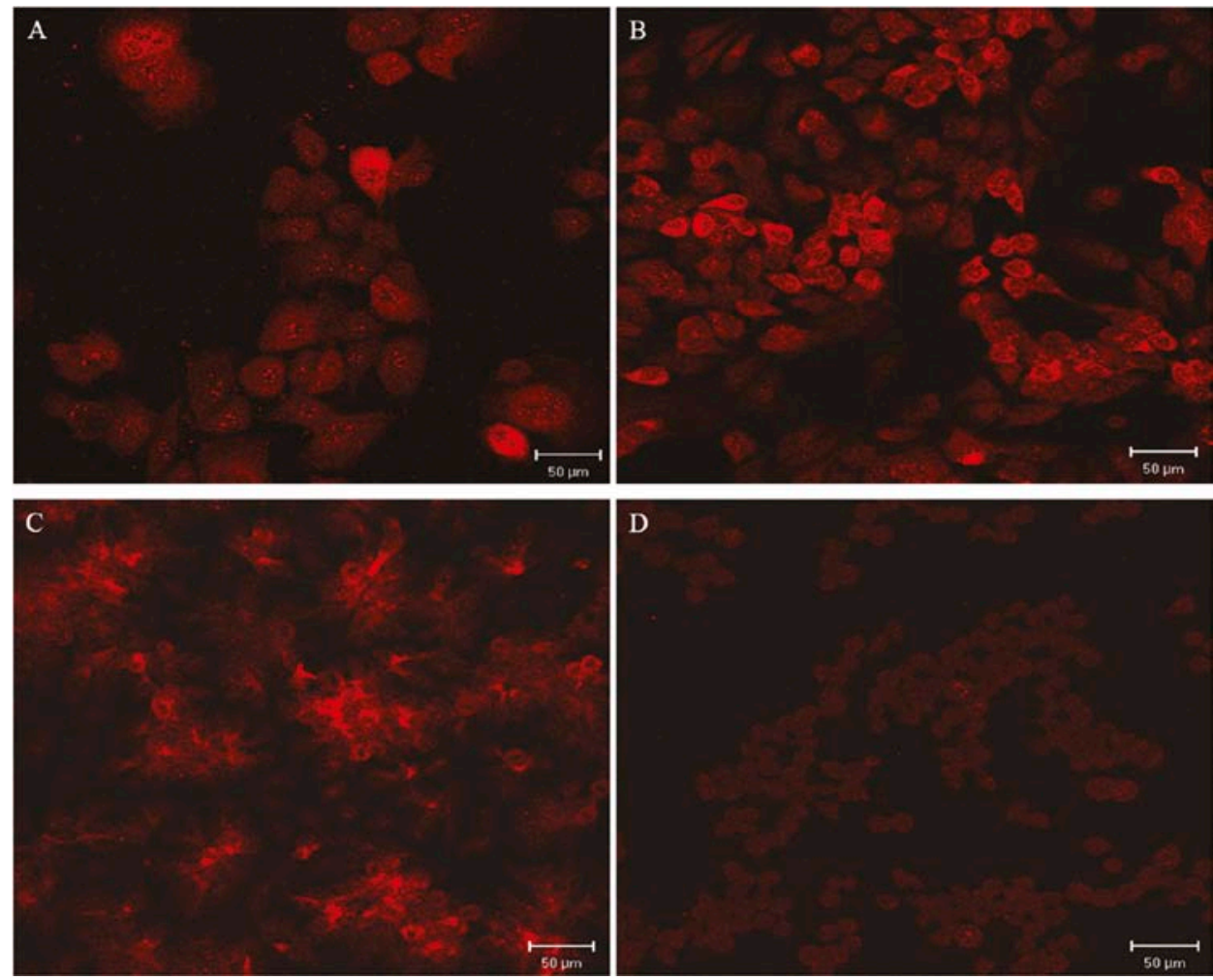

Figure 2. Immunofluorescence staining of CFH protein in lung cancer cell lines. Positive staining of CFH in H226 (A), H2228 (B) and H2170 (C) (25-fold magnification) is associated with cytoplasm and membrane. In cell line COLO677 (D) the CFH staining is negative (25-fold magnification).
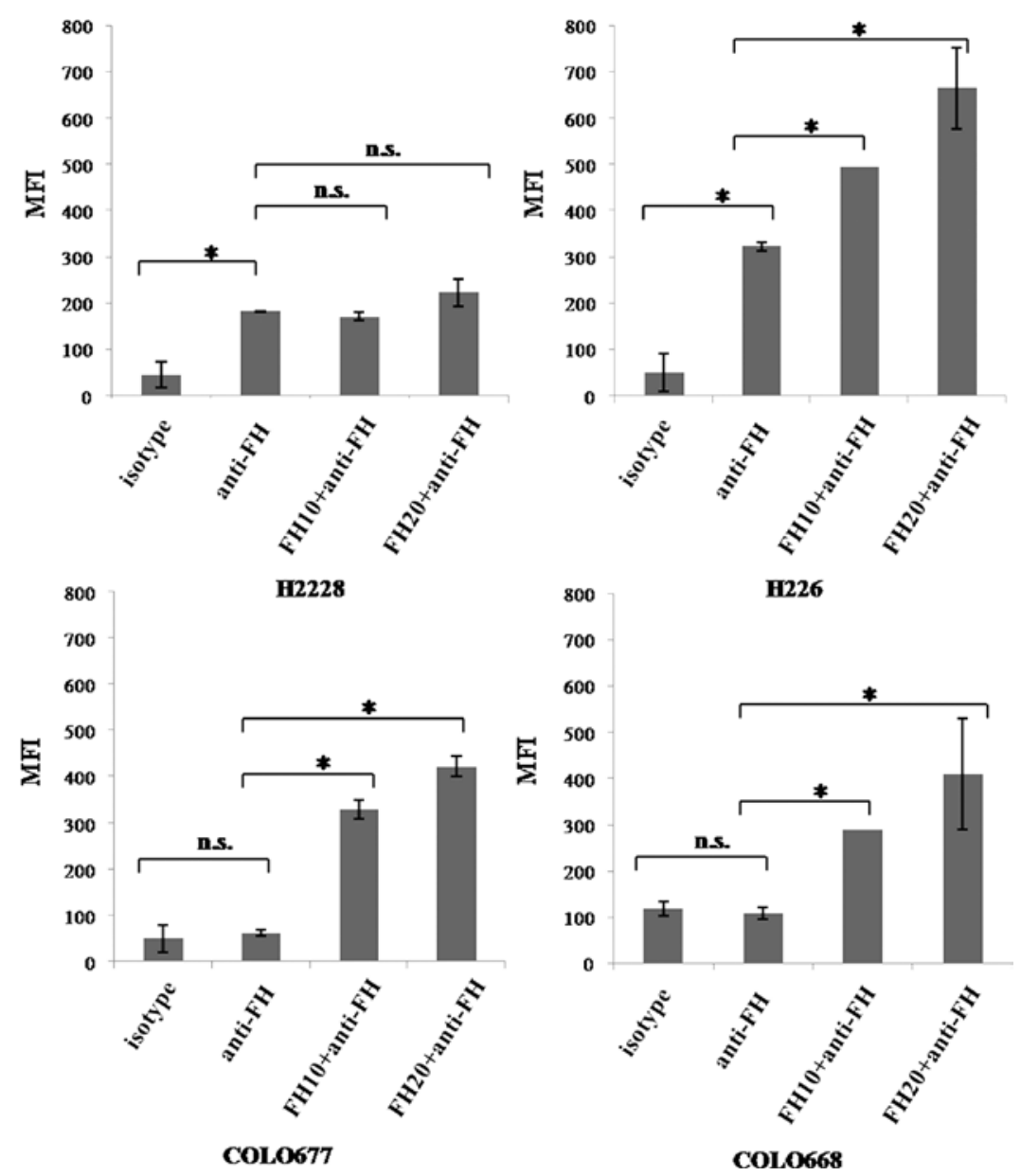

Figure 3. CFH binds to lung cancer cells. CFH binding was determined by flow cytometry. $\mathrm{H} 226$ and $\mathrm{H} 2228$ bind both endogenous and exogenous CFH, COLO677 and COLO668 bind only exogenous CFH. MFI: median fluorescent intensity; bar: \pm SE; "p $<0.05$; n.s.: not significant. 
A

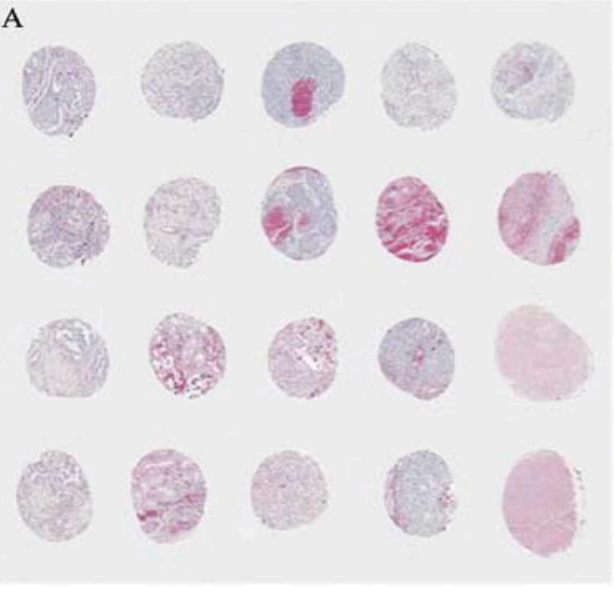

C

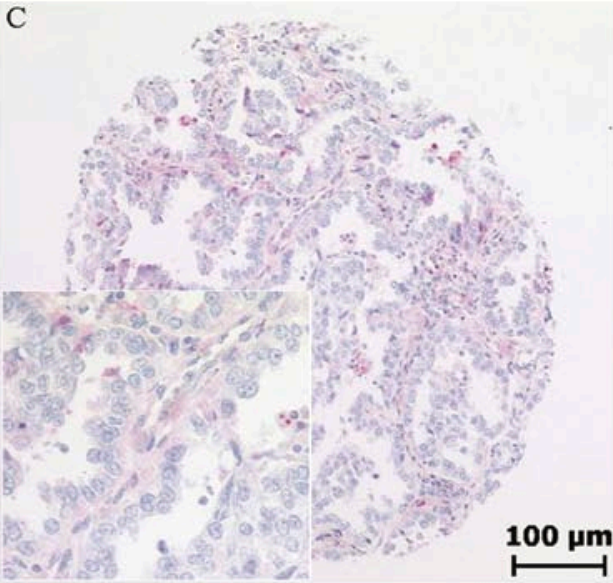

E

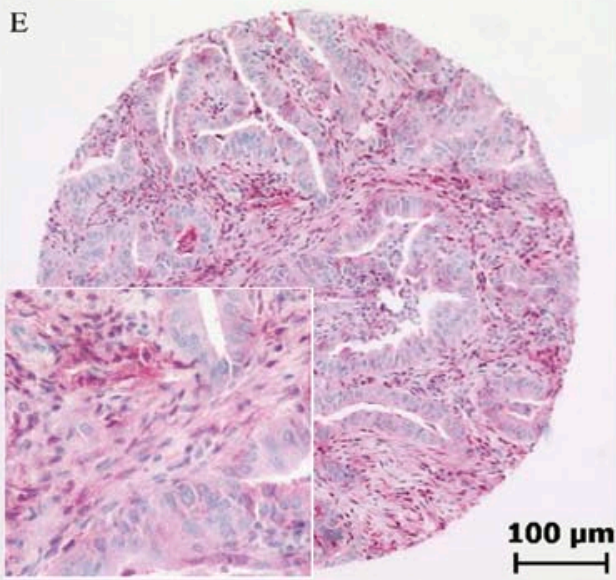

B

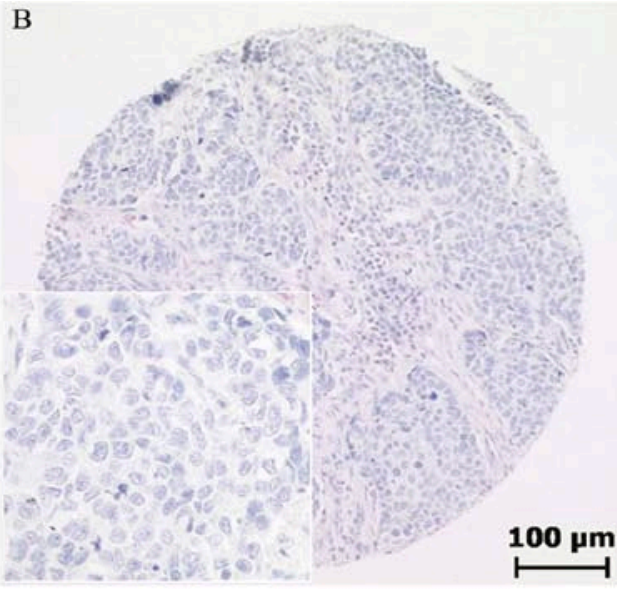

D
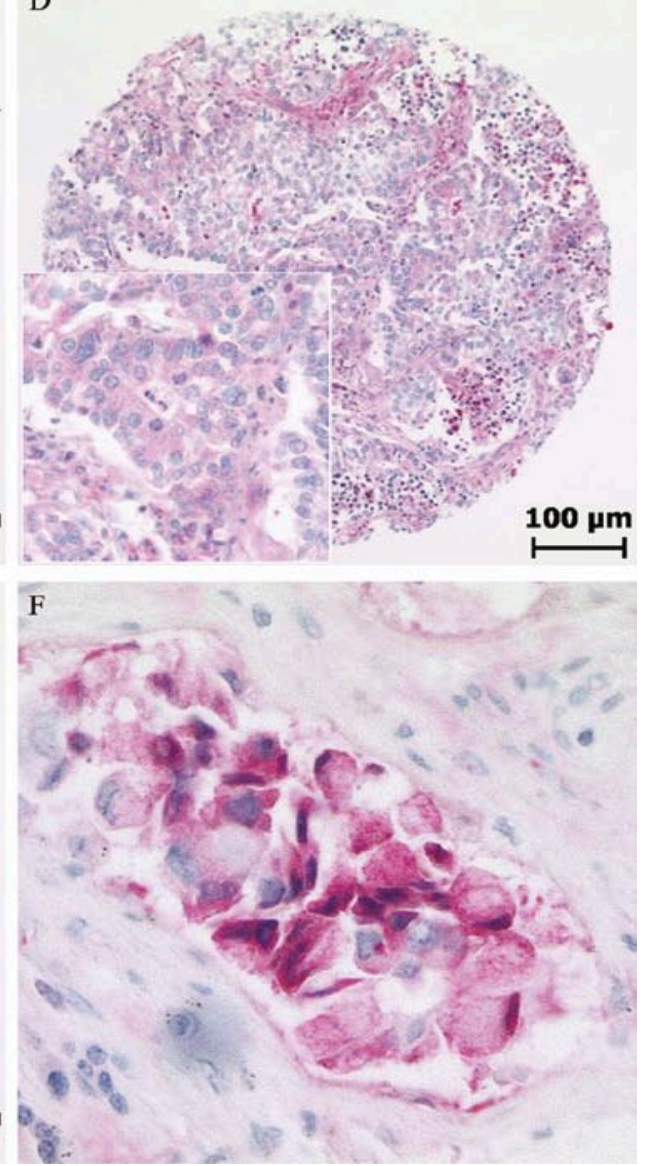

Figure 4. CFH protein expression in primary lung tumors. (A) A section from TMA (12.5-fold magnification); (B) CFH negative; (C) CFH weakly positive; (D) $\mathrm{CFH}$ moderately positive; (E) CFH strongly positive. (F) Apoptotic cells showed increased expression of CFH (50-fold magnification).

protein expression in primary lung tumors. One section is shown in Fig. 4A. CFH expression was highest in infectious cells including macrophage cells, but was also detectable at lower intensity in smooth muscle cells of the lung vessels and blood serum. In tumor samples, as shown in Table I, 54 out of 101 cases $(53.5 \%)$ were positive for $\mathrm{CFH}$ with either a membrane-like pattern or granular cytoplasmic staining, while other 47 cases (46.5\%) exhibited no expression. Examples of CFH staining are present in Fig. 4B-E. In apoptotic cells, enhanced factor $\mathrm{H}$ expression could be observed (Fig. 4F). In normal lung epithelium, $\mathrm{CFH}$ staining could not be detected (data not shown). The expression of CFH did not differ significantly by tumor grade, tumor stage, lymph node status, age and sex (data not shown), however, the difference of $\mathrm{CFH}$ expression between squamous cell carcinomas (SCCs) and adenocarcinomas (ADCs) reached statistical significance with ADCs showing more frequently positive expression of $\mathrm{CFH}$ compared to SCCs $(\mathrm{p}=0.009$, Table II).

On analysis of 59 patients with primary lung cancer, from follow up data we found that the median survival time was 23 months (range 2-66 months), and there was no significant effect of $\mathrm{CFH}$ protein expression on disease-free survival 
Table I. Study cohort.

\begin{tabular}{lcc}
\hline & CFH-negative n $(\%)$ & CFH-positive $\mathrm{n}(\%)$ \\
\hline No $(\mathrm{n}=101)$ & $47(46.5)$ & $54(53.5)$ \\
SCC & $25(24.8)$ & $16(15.8)$ \\
LCLC & $2(2.0)$ & $3(3.0)$ \\
ADC & $18(17.8)$ & $35(34.7)$ \\
SCLC & $2(2.0)$ & $0(0.0)$ \\
Grade 1-2 & $30(31.3)$ & $27(28.1)$ \\
Grade 3 & $14(14.6)$ & $25(26.0)$ \\
pT1 & $10(10.2)$ & $10(10.2)$ \\
pT2 & & $42(42.9)$ \\
pN0 & $36(36.7)$ & $30(31.3)$ \\
pN1 ${ }^{+}$ & $22(22.9)$ & $22(22.9)$
\end{tabular}

ADC, adenocarcinoma; SCC, squamous cell carcinoma; LCLC, large cell lung carcinoma; SCLC, small cell lung carcinoma; $\mathrm{pT}^{+}=\mathrm{pT} 2-\mathrm{pT} 4$; $\mathrm{pN} 1^{+}=\mathrm{pN} 1-\mathrm{pN} 3$.

Table II. Expression of CFH in patients with ADC and SCC.

\begin{tabular}{lccc}
\hline & CFH (-) & CFH (+) & p-value $^{\mathrm{a}}$ \\
\hline ADC & $18(19.1 \%)$ & $35(37.3 \%)$ & 0.009 \\
SCC & $25(26.6 \%)$ & $16(17.0 \%)$ & 0.009 \\
\hline
\end{tabular}

ap-value was calculated by Chi-square test.

Table III. Correlation between CFH expression and survival time in patients with ADC.

\begin{tabular}{lrrr}
\hline Survival time & CFH (-) & CFH (+) & p-value $^{\mathrm{a}}$ \\
\hline$\leq 20$ months & $7(20.6 \%)$ & $6(17.6 \%)$ & 0.033 \\
$>20$ months & $19(55.9 \%)$ & $2(5.9 \%)$ & 0.033 \\
\hline
\end{tabular}

${ }^{a}$ p-value was calculated by Fisher's exact test.

( $\mathrm{p}=0.128$, Kaplan-Meier statistical analysis, Fig. 5A). In the 36 patients with ADC, there was a tendency that tumors with CFH-positive staining had a worse prognosis compared to tumors with negative staining of $\mathrm{CFH}$ ( $\mathrm{p}=0.082$, KaplanMeier statistical analysis, Fig. 5B). Interestingly, ADCs with shorter survival time ( $<20$ months) had stronger CFH staining $(\mathrm{p}=0.033$, Table III $)$.

\section{Discussion}

The major findings present in the current study can be summarized as follows: i) $\mathrm{CFH}$ was expressed in some of non-small cell lung cancer cell lines, ii) $\mathrm{CFH}$ was not expressed in small
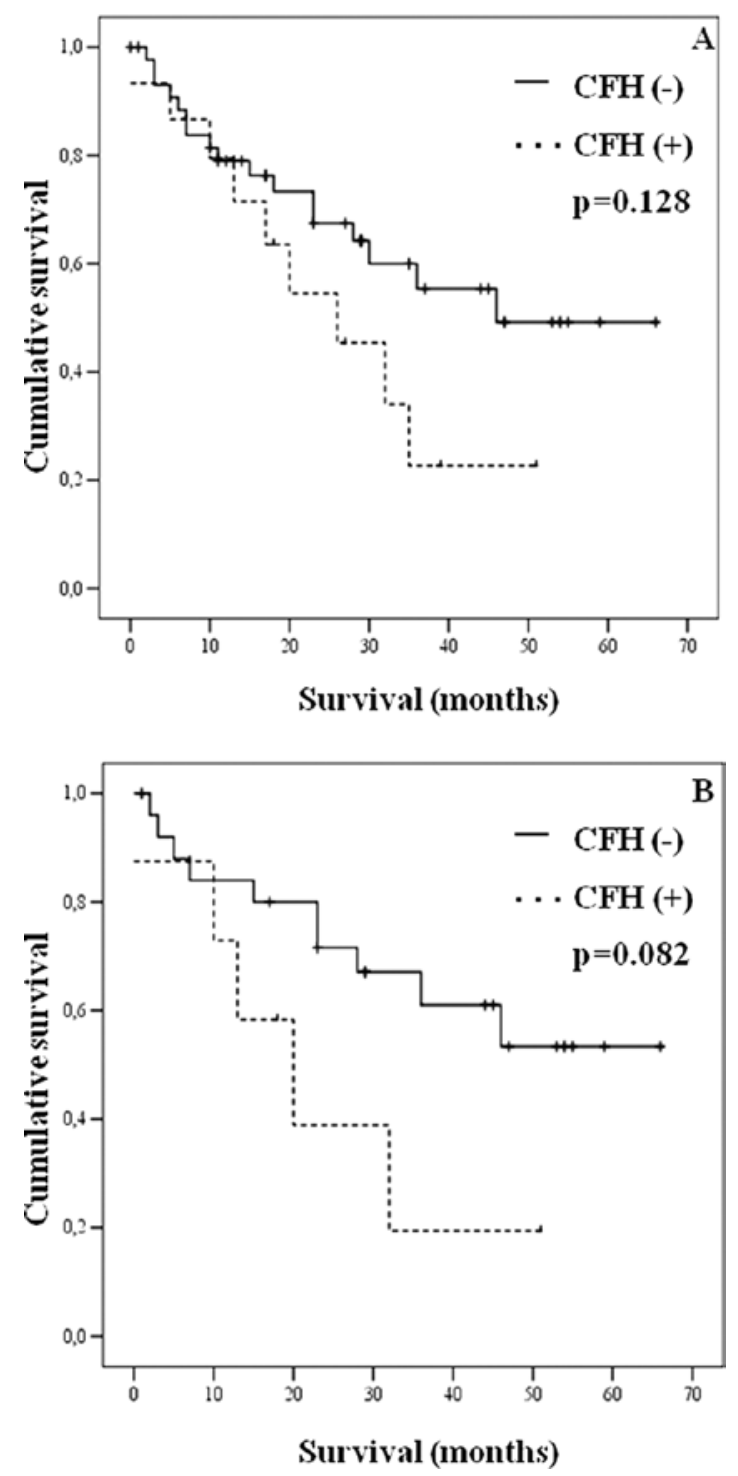

Figure 5. Kaplan-Meier survival curves of high CFH expression versus low $\mathrm{CFH}$ expression in patients with adenocarcinoma showed a tendency that high expression of CFH is correlated with poor prognosis. (A) In NSCLC, (B) in adenocarcinoma of the lung.

cell lung cancer cell lines, iii) cancer cells bound $\mathrm{CFH}$ to their surface, iv) the majority of tumor samples $(53.5 \%)$ exhibited CFH positive staining, v) CFH could be a biomarker for adenocarcinoma of the lung. This is the first report that $\mathrm{CFH}$ could distinguish ADC from SCC of the lung.

$\mathrm{CFH}$, a single polypeptide chain glycoprotein $(150-\mathrm{kDa})$ present in plasma, acts as the most prominent fluid-phase complement inhibitor (16). This central fluid phase protein regulates the alternative pathway of the complement system (17-19). CFH was expressed in various malignant cell lines including ovarian, colon, bladder, liver and lung cancer cell lines $(6,10,20-22)$. It binds to C3b and blocks the alternative pathway of complement by i) acting as cofactor for $\mathrm{C} 3 \mathrm{~b}$ cleavage, ii) accelerating the decay of the alternative $\mathrm{C} 3$ convertase, and iii) preventing binding of factor $\mathrm{B}$ to $\mathrm{C} 3$. By inhibiting alternative complement activity, factor $\mathrm{H}$ also protects tumor cells against immune attack (23-25). The utility of CFH as a diagnostic marker for transitional cell cancer of the bladder 
raised the question whether it could be a marker for other malignancies.

In our study, we analysed the mRNA and protein expression of CFH in a panel of lung cancer cell lines by RT-PCR, Western blotting, flow cytometry and immunofluorescence analysis. It turned out that several human NSCLC cell lines constitutively expressed and secreted CFH, while in SCLC cell lines, no $\mathrm{CFH}$ expression was detectable. This result is in line with previous report by Ajona et al (12), who found that neither SCLC nor carcinoid cell lines expressed $\mathrm{CFH}$, suggesting a phenotypic correlation between the expression of $\mathrm{CFH}$ and the neuroendocrine differentiation of the tumors. We further analyzed the ability of factor $\mathrm{H}$ binding. It turned out that factor $\mathrm{H}$ binding to the cell surface is independent of its expression in tumor cells.

In a survey of protein expression of CFH in 101 primary lung tumors, we found that more than half of the samples $(53.5 \%)$ showed CFH-positive staining, while the other tumor samples were negatively stained, indicating that high expression of $\mathrm{CFH}$ is not an infrequent feature of this type of cancer. We could only divide the primary tumors into two major subgroups of ADC and SCC for statistical analysis, since the sample size of small cell lung cancer (SCLC) and large cell lung cancer (LCLC) was too small to be further evaluated. Although expression of CFH was not significantly associated with tumor progression, differentiation and lymph node metastasis, it was significantly higher in ADCs than in SCCs $(\mathrm{p}<0.01)$. The protein expression of $\mathrm{CFH}$ has previously only been evaluated in patients with primary colon adenocarcinoma by Wilczek et al. They found that CFH was expressed in both primary colon adenocarcinoma and metastatic foci in liver (6). It is not yet clear if overexpression of $\mathrm{CFH}$ is a common event in adenocarcinoma, nevertheless, our result suggests that $\mathrm{CFH}$ could be a novel marker to distinguish ADC from SCC of the lung.

Additionally, consistent with previous observations that CFH binds to apoptotic and necrotic cells $(26,27)$, we found that apoptotic cells expressed more $\mathrm{CFH}$ compared to nonapoptotic cells of primary lung tumors. Most apoptotic cells do not undergo complement-mediated lysis probably due to overexpression of $\mathrm{CFH}$, which compensates for the loss of $\mathrm{m}-\mathrm{C}$-Reg, a membrane-bound complement regulatory protein, and protect against excessive complement activation and lysis (28).

It has been reported that some of human non-small cell lung cancer cell lines highly expressing $\mathrm{CFH}$ or $\mathrm{CFH}-$ like protein are more susceptible to complement-mediated damage and more efficiently deposited $\mathrm{C} 3 \mathrm{~b}$ on the cell surface when they were blocked by specific anti-factor $\mathrm{H}$ antibodies (12). Tumor cells that express and bind $\mathrm{CFH}$ to their surface can prevent $\mathrm{C} 3 \mathrm{~b}$ accumulation upon their cell membranes and lead to high resistance of these cells to complement-mediated lysis (12). Based on the characteristics, we supposed that tumors with high expression of $\mathrm{CFH}$ could be more aggressive and have a worse clinical outcome. To test this hypothesis, we analysed the effect of CFH expression on patient survival. Again, in adenocarcinoma of the lung, there was a tendency that $\mathrm{CFH}$ positive-tumors had a worse clinical outcome compared to $\mathrm{CFH}$ negative-tumors, although it did not reach statistical significance. Patients with ADC who had positive expression of $\mathrm{CFH}$ ended with a shorter survival time $(<20$ months). Evidence is not sufficient to prove the role of $\mathrm{CFH}$ as a prognostic marker in adenocarcinoma of the lung. Future studies based on the investigation of more tumor samples are needed to warrant the prognostic role of CFH in lung cancer.

In summary, non-small cell lung cancer expressed $\mathrm{CFH}$, and $\mathrm{CFH}$ might be a diagnostic marker for adenocarcinoma of the lung. For a long time, lung cancer was not considered an immune-sensitive malignancy. Although there is increasing evidence that NSCLC and SCLC can evoke specific humeral and cellular anti-tumor immune responses, lung cancer immunotherapy lags behind similar efforts in melanoma, renal cell and prostate cancer. Our results could provide further insight into understanding of pathogenesis and could have implication for the design of more effective immunotherapeutic strategies for this fatal disease.

\section{Acknowledgments}

The work was supported by the Deutsche Krebshilfe (grant no. 108003).

\section{References}

1. Gazdar AF, Shigematsu H, Herz J and Minna JD: Mutations and addiction to EGFR: the Achilles heal of lung cancers? Trends Mol Med 10: 481-486, 2004.

2. Copin MC, Buisine MP, Devisme L, et al: Normal respiratory mucosa, precursor lesions and lung carcinomas: differential expression of human mucin genes. Front Biosci 6: 1264-1275, 2001.

3. Jemal A, Siegel R, Ward E, et al: Cancer statistics. CA Cancer J Clin 58: 71-96, 2008.

4. Kargi A, Gurel D and Tuna B: The diagnostic value of TTF-1, CK 5/6, and p63 immunostaining in classification of lung carcinomas. Appl Immunohistochem Mol Morphol 15: 415-420, 2007.

5. Varsano S, Rashkovsky L, Shapiro H, Ophir D and MarkBentankur T: Human lung cancer cell lines express cell membrane complement inhibitory proteins and are extremely resistant to complement-mediated lysis; a comparison with normal human respiratory epithelium in vitro, and an insight into mechanism(s) of resistance. Clin Exp Immunol 113: 173-182, 1998.

6. Wilczek E, Rzepko R, Nowis D, et al: The possible role of factor $\mathrm{H}$ in colon cancer resistance to complement attack. Int J Cancer 122: 2030-2037, 2008.

7. Harboe M and Mollnes TE: The alternative complement pathway revisited. J Cell Mol Med 12: 1074-1084, 2008.

8. Zipfel PF and Skerka C: FHL-1/reconectin: a human complement and immune regulator with cell-adhesive function. Immunol Today 20: 135-140, 1999.

9. Gasque P, Thomas A, Fontaine M and Morgan BP: Complement activation on human neuroblastoma cell lines in vitro: route of activation and expression of functional complement regulatory proteins. J Neuroimmunol 66: 29-40, 1996.

10. Junnikkala S, Jokiranta TS, Friese MA, Jarva H, Zipfel PF and Meri S: Exceptional resistance of human $\mathrm{H} 2$ glioblastoma cells to complement mediated killing by expression and utilization of factor $\mathrm{H}$ and factor H-like protein1. J Immunol 164: 6075-6081, 2000.

11. Junnikkala S, Hakulinen J, Jarva H, et al: Secretion of soluble complement inhibitors factor $\mathrm{H}$ and factor $\mathrm{H}$-like protein (FHL-1) by ovarian tumor cells. Br J Cancer 87: 1119-1127, 2002.

12. Ajona D, Castaño Z, Garayoa M, et al: Expression of complement factor $\mathrm{H}$ by lung cancer cells: effects on the activation of the alternative pathway of complement. Cancer Res 64: 6310-6318, 2004.

13. Kinders R, Jones T, Root R, et al: Complement factor $\mathrm{H}$ or a related protein is a marker for transitional cell cancer of the bladder. Clin Cancer Res 4: 2511-2520, 1998.

14. Berndt A, Borsi L, Hyckel P and Kosmehl H: Fibrillary co-deposition of laminin-5 and large unspliced tenascin- $\mathrm{C}$ in the invasive front of oral squamous cell carcinoma in vivo and in vitro. $\mathrm{J}$ Cancer Res Clin Oncol 127: 286-292, 2001. 
15. Knösel T, Yu Y, Stein U, et al: Overexpression of cyclooxygenase-2 correlates with chromosomal gain at the cyclooxygenase-2 locus and decreased patient survival in advanced colorectal carcinomas. Dis Colon Rectum 47: 70-77, 2004.

16. Friese MA, Hellwage J, Jokiranta TS, et al: Different regulation of factor $\mathrm{H}$ and $\mathrm{FHL}-1$ /reconectin by inflammatory mediators and expression of the two proteins in rheumatoid arthritis (RA). Clin Exp Immunol 121: 406-415, 2000.

17. Whaley $\mathrm{K}$ and Ruddy S: Modulation of $\mathrm{C} 3 \mathrm{~b}$ hemolytic activity by a plasma protein distinct from C3b inactivator. Science 193: 1011-1013, 1976.

18. Harrison RA and Lachmann PJ: The physiological breakdown of the third component of human complement. Mol Immunol 17: 9-20, 1980

19. Fearon DT and Austen KF: Activation of the alternative complement pathway due to resistance of zymosan-bound. Proc Natl Acad Sci USA 74: 1683-1687, 1977.

20. Cheng ZZ, Corey MJ, Pärepalo M, et al: Complement factor $\mathrm{H}$ as a marker for detection of bladder cancer. Clin Chem 51: 856-863, 2005

21. Schlaf G, Beisel N, Pollok-Kopp B, Schieferdecker H, Demberg T and Götze O: Constitutive expression and regulation of rat complement factor $\mathrm{H}$ in primary cultures of hepatocytes, Kupffer cells, and two hepatoma cell lines. Lab Invest 82: 183-192, 2002.

22. Ajona D, Hsu YF, Corrales L, Montuenga LM and Pio R: Downregulation of Human complement factor $\mathrm{H}$ sensitizes non-small cell lung cancer cells to complement attack and reduces in vivo tumor growth. J Immunol 178: 5991-5998, 2007.
23. Rodríguez de Córdoba S, Esparza-Gordillo J, Goicoechea de Jorge E, Lopez-Trascasa M and Sánchez-Corral P: The human complement factor $\mathrm{H}$ : functional roles, genetic variations and disease associations. Mol Immunol 41: 355-367, 2004.

24. Sim E, Wood AB, Hsiung LM and Sim RB: Pattern of degradation of human complement fragment, C3b. FEBS Lett 132: 55-60, 1981.

25. Gasque P, Julen N, Ischenko AM, et al: Expression of complement components of the alternative pathway by glioma cell lines. J Immunol 149: 1381-1387, 1992.

26. Leffler J, Herbert AP, Norström E, et al: Annexin-II, DNA, and histones serve as factor $\mathrm{H}$ ligands on the surface of apoptotic cells. J Biol Chem 285: 3766-3776, 2010.

27. Mihlan M, Stippa S, Józsi M and Zipfel PF: Monomeric CRP contributes to complement control in fluid phase and on cellular surfaces and increases phagocytosis by recruiting factor $\mathrm{H}$. Cell Death Differ 16: 1630-1640, 2009.

28. Trauw LA, Bengtsson AA, Gelderman KA, Dahlbäck B, Sturfelt $\mathrm{G}$ and Blom AM: C4b-binding protein and factor $\mathrm{H}$ compensate for the loss of membrane-bound complement inhibitors to protect apoptotic cells against excessive complement attack. J Biol Chem 282: 28540-28548, 2007. 